\title{
Perfil de sensibilidad y resistencia de Staphylococcus aureus. Experiencia en el Hospital Nacional Hipólito Unanue
}

\author{
Edgardo Mamani ${ }^{1}$, Daniel Luján ${ }^{2}$, Giovanni Pajuelo ${ }^{2}$
}

\begin{abstract}
Resumen
Objetivo: Documentar la susceptibilidad de Staphylococcus aureus a diversos antimicrobianos, entre pacientes hospitalizados y ambulatorios. Diseño: Análisis retrospectivo. Lugar: Hospital Nacional Hipólito Unanue, hospital universitario. Materiales: Entre enero y diciembre de 2003, se estudió la sensibilidad y resistencia a antibióticos de cepas de Staphylococcus aureus provenientes de vías respiratorias. Intervenciones: Se empleó el método de Kirby-Bauer, en el medio de Mueller - Hinton, con las recomendaciones del CLSI. Principal medida de resultados: Sensibilidad a antibióticos. Resultados: Se aislóy evaluó 217 cepas de Staphylococcus aureus provenientes de vías respiratorias. La resistencia encontrada en cepas provenientes de pacientes hospitalizados a oxacilina fue de 32\%, a gentamicina 35\% y a ciprofloxacina $58 \%$ y se registró un $100 \%$ de sensibilidad a vancomicina. Conclusión: Se encontró una resistencia baja a oxacilina.
\end{abstract}

Palabrasclave Staphylococcus aureus; oxacilina; resistencia microbiana a las drogas.

Staphylococcus aureus sensitivity and resistance profile. Experience at Hipolito Unanue National Hospital

\begin{abstract}
Objective: The determine Staphylococcus aureus susceptibility to different antimicrobians in both inpatients and outpatients. Design: Retrospective analysis. Setting: Hipolito Unanue National Hospital, a teaching hospital. Materials: From January through December 2003 we determined sensitivity and resistance of Staphylococcus aureus strains obtained from the respiratory tract. Interventions: We used the Kirby-Bauer method in the Bacto Mueller Hinton Medium following the CLSI recommendations. Main outcome measures: Sensitivity to antibiotics. Results: Two hundred and seventeen Staphylococcus aureus strains originated from the respiratory tract were isolated. Oxacillin resistance in inpatiens was found in $32 \%$ of strains, to gentamicin $35 \%$, and to ciprofloxacin $58 \%$, and $100 \%$ sensitivity to vancomycin. Conclusion: Low resistance to oxacillin was found.
\end{abstract}

Key words: Staphylococcus aureus, oxacillin; drug resistance, microbial.

Ministerio de Salud, Dirección de Salud V. Lima, Perú.

2 Universidad Nacional Federico Villarreal. Lima, Perú.

\section{INTRODUCCIÓN}

Staphylococcus aureus es el mayor patógeno humano causante de infecciones a la piel y tejidos, neumonía, septicemia e infecciones por dispositivos asociados. La emergencia de cepas resistentes a meticilina $\mathrm{y}$ otros agentes antibacteriales ha llegado a ser una preocupación mayor especialmente en el ambiente hospitalario, esto es por la alta mortalidad debido a las infecciones sistémicas ocasionadas por Staphylococcus aureus meticilino resistente (SAMR) ${ }^{(1-3)}$.

Naturalmente, un entendimiento de la dinámica de la diseminación y una identificación de transmisiones o brotes son de interés no solo para los epidemiólogos de la salud pública sino también para microbiólogos clínicos involucrados en manejo de pacientes en una labor diaria $\left({ }^{4}\right)$.

Algunas investigaciones sugieren que las infecciones por SAMR están asociadas con 
hospitalizaciones prolongadas, mortalidad incrementada y costos elevados, comparados con infecciones debidas a $S$. aureus susceptible a meticilina. Sin embargo, tales comparaciones pueden ser confundidas con una incrementada incidencia de condiciones comórbidas entre pacientes con infección por SAMR $\left(^{5,6}\right)$. Infortunadamente, las opciones terapéuticas para pacientes con infecciones SAMR son limitadas; la opción primaria es terapia con vancomicina intravenosa, ya que otros antimicrobianos, incluyendo las fluoroquinolonas y cefalosporinas de tercera generación, son inefectivas contra SAMR ${ }^{(7)}$. La emergencia de $S$. aureus con resistencia intermedia a glicopéptidos y SAMR heterorresistente sugiere que la resistencia completa a glicopéptidos puede desarrollarse rápidamente y limitar la utilidad de vancomicina, lo cual subraya la necesidad de nuevos antibióticos $\left({ }^{8,9}\right)$.

Es el propósito de este estudio realizar una evaluación del estado actual de los patrones de resistencia de esta bacteria en un hospital local y contribuir de esta manera a su estudio epidemiológico.

\section{MATERIALES Y MÉTODOS}

Este estudio incluyó pacientes hospitalizados y ambulatorios que utilizaron los servicios de salud del Hospital Nacional Hipólito Unanue, entre el $1^{\circ}$ de enero y 31 de diciembre de 2003, cuyas muestras de vía respiratoria fueron remitidas al laboratorio de Microbiología del Hospital Nacional Hipólito Unanue para el cultivo correspondiente. Se tomó en cuenta una muestra por paciente. No se consideró sintomatología, sexo o edad.

Se realizó la siembra en placas de agar sangre y se incubó a $37{ }^{\circ} \mathrm{C}$ por 24 horas. Se consideró cultivos positivos aquellos en los que al menos pudo observarse una colonia sugerente de $S$. aureus, en algún cuadrante de la placa de cultivo.
La identificación se llevó a cabo por las características del cultivo, observación de colonias de color blanco o dorado, circundadas por un halo de hemólisis, coloración de Gram, pruebas de catalasa y coagulasa en tubo.

Las cepas aisladas de Staphylococcus aureus fueron analizadas con el método de difusión en disco de Kirby - Bauer, en el medio de Muller - Hinton, y siguiendo las recomendaciones del Clinical and Laboratory Standards Institute (CLSI) (ex NCCLS). Se utilizó una cepa de referencia, para el control de calidad interno, $S$. aureus ATCC 25923.

\section{RESULTADOS}

Se incluyó 217 cepas de Staphylococcus aureus para su evaluación.

En cuanto al análisis de susceptibilidad antimicrobiana registrado, se obtuvo un patrón de resistencia en pacientes hospitalizados de $32 \%$ a oxacilina, $35 \%$ a gentamicina, $10 \%$ a amikacina, $58 \%$ a ciprofloxacina; y, con relación a la sensibilidad, se obtuvo $100 \%$ a vancomicina. En cuanto a los pacientes ambulatorios, se obtuvo porcentajes de resistencia de $21 \%$ a oxacilina, $32 \%$ a gentamicina, $8 \%$ a amikacina, $52 \%$ a ciprofloxacina; y se obtuvo $100 \%$ de sensibilidad a vancomicina (Tabla 1).

Tabla 1. Análisis de susceptibilidad antimicrobiana en cepas de Staphylococcus aureus aisladas en el Hospital Nacional Hipólito Unanue, durante el año 2003.

\begin{tabular}{lrcrr}
\hline & \multicolumn{2}{c}{ Hospitalizados } & \multicolumn{2}{c}{ Ambulatorios } \\
Antimicrobiano & ${ }^{*} \mathrm{~S}(\%)$ & $* * \mathrm{R}(\%)$ & ${ }^{*} \mathrm{~S}(\%)$ & $* * \mathrm{R}(\%)$ \\
\hline Oxacilina & 68 & 32 & 79 & 21 \\
Gentamicina & 65 & 35 & 68 & 32 \\
Amikacina & 90 & 10 & 92 & 8 \\
Ciprofloxacina & 42 & 58 & 48 & 52 \\
Vancomicina & 100 & 0 & 100 & 0 \\
\hline
\end{tabular}

${ }^{*} S=$ Sensible

${ }^{* *} R=$ Resistente 


\section{DISCUSIÓN}

La importancia y el incremento de los brotes por SAMR han sido reconocidos en muchos países. Existe una búsqueda por nueva y mejor información respecto al mecanismo de diseminación local que pueda proveer herramientas para controlar un mayor incremento de SAMR. Es importante determinar si las cepas específicas resistentes a meticilina causan la mayoría de infecciones por Staphylococcus aureus en los hospitales $\left({ }^{10}\right)$.

Con la búsqueda y actitud para acabar con estas cepas, una gran parte del problema por SAMR puede ser controlada. Debe trabajarse ordenadamente para restringir la transmisión de SAMR en el medio ambiente hospitalario; se debe tomar estrictas medidas de higiene, acompañadas con una acuciosa tipificación de cepas $\left({ }^{4}\right)$.

Se ha estudiado las asociaciones de SAMR a infecciones de heridas quirúrgicas, determinándose un $9,8 \%$ de prevalencia en aislados nosocomiales, en EE UU ${ }^{(11)}$. Asimismo, se ha determinado la epidemiología de estas infecciones en unidades de riesgo neonatal alto, obteniéndose un $22 \%$ de aislados del personal de salud en Venezuela ${ }^{(12)}$. También, se ha estudiado la bacteriemia por SAMR, encontrándo-

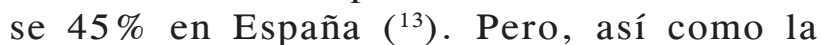
epidemiología de SAMR aislados en centros nosocomiales ha sido bien estudiada, poco se conoce acerca de la diseminación de SAMR entre la comunidad circundante a los hospitales. Un estudio en EE UU encontró una alta relación entre pacientes hospitalizados y ambulatorios, con porcentajes de $45,7 \%$ y $28,6 \%$ de aislamientos, respectivamente $\left({ }^{14}\right)$. Asimismo, se ha encontrado diseminación por clones específicos en áreas geográficas de baja incidencia de SAMR (parte occidental de Suiza), sugiriéndose que el origen clonal de estos aislados fue por resistencia heterogénea a meticilina $\left({ }^{15}\right)$.
Se ha explicado los aspectos relacionados a la resistencia en SAMR, determinándose que la mayoría de aislados presenta una heterorresistencia, es decir, una resistencia heterogénea a diferentes antimicrobianos y se ha comparado diferentes métodos para determinar SAMR $\left({ }^{16}\right)$. Por otra parte, los mecanismos de la resistencia a diferentes antimicrobianos también ha sido motivo de estudio $\left({ }^{17-20}\right)$, así como la frecuencia de sus mutaciones $\left({ }^{21}\right)$.

En este trabajo, se eligió como cepa multirresistente a aquel aislado que presentara resistencia a por lo menos tres antimicrobianos. En cuanto a la resistencia detectada para meticilina/oxacilina, se encontró valores de $32 \%$ en pacientes hospitalizados, cifra menor en relación con estudios realizados en hospitales de Lima y Callao. Es así como, en el hospital Daniel Alcides Carrión del Callao se halló 36\% $\left({ }^{22}\right)$, y en hospitales de Lima, Arzobispo Loayza 53,6\% (23), Guillermo Almenara 90\% ${ }^{(24)}$, Edgardo Rebagliati $85 \%\left({ }^{25}\right)$ e Instituto de Enfermedades Neoplásicas, $100 \%$ de resistencia $\left({ }^{26}\right)$. En el hospital del Sur de Arequipa se obtuvo $71,4 \%\left({ }^{27}\right)$. En un primer estudio, en el hospital Regional Honorio Delgado, utilizando difusión en disco y dilución en agar, se determinó $66,7 \%\left({ }^{28}\right)$; pero, posteriormente y, utilizando estos mismos métodos microbiológicos, se registró $32,9 \%$, indicándose además susceptibilidad borderline de $19,7 \%\left({ }^{29}\right)$. La menor resistencia hallada en este estudio a oxacilina podría explicarse por carecer estas cepas del gen mecA y lo que vendría a determinar su patrón de resistencia alterno mediado por mutaciones de las PBP (penicillin binding proteins) normales, también estaría disminuido.

Los aminoglicósidos registraron entre $10 \%$ y $35 \%$ de resistencia, tomando gentamicina el valor más alto. La resistencia se debe a que estos antimicrobianos son modificados por medio de acetilación, adenilación o fosforilación y por lo tanto 
no pueden unirse adecuadamente a los ribosomas y desde allí impedir la biosíntesis normal de proteínas de Staphylococcus aureus $\left({ }^{30}\right)$.

Las fluoroquinolonas presentaron un patrón de resistencia de $58 \%$, resultado de una alteración en el codón 84 o en el codón 85 en la ADN girasa bacteriana y de esta manera se altera el sitio de acción del antimicrobiano $\left({ }^{31}\right)$.

Los glicopéptidos mostraron $100 \%$ de sensibilidad, correspondiente con lo reportado a nivel local. En cuanto a la resistencia a los glicopéptidos, lo que en un principio fue preocupante con la aparición de cepas de Staphylococcus aureus con resistencia intermedia a vancomicina (SAIV) $\left({ }^{32,33}\right)$, ha sido confirmado con la aparición de una cepa $100 \%$ resistente a vancomicina (SARV), en el año 2002, en los EE UU $\left({ }^{34}\right)$. Esta cepa portaba el gen VanA de resistencia a vancomicina en enterococo, sugiriendo que la resistencia pudo ser adquirida por intercambio de material genético con la cepa de Enterococcus faecalis resistente a vancomicina (ERV), de la cual también era portador el paciente.

En conclusión, si bien es cierto que el porcentaje de resistencia para oxacilina ha sido ligeramente bajo, favoreciendo de este modo las estrategias que se podría utilizar para controlar y aminorar la diseminación intrahospitalaria del $S$. aureus multirresistente, los reportes internacionales nos advierten la urgente necesidad de aplicarlas, ya que estas cepas podrían tornarse resistentes a vancomicina y nuevamente existiría la amenaza de que las infecciones estafilocócicas puedan llegar a ser intratables.

\section{REFERENCIAS BIBLIOGRÁFICAS}

1. Berger-Bächi B. Update on methicillin resistance mechanism in Staphylococci. Chemotherapy. 1996;42(suppl 2):19-26.
2. Kayser F. Basic aspects of antibiotic resistance in the multiresistant Staphylococcus: an overview. Chemotherapy. 1996;42 (suppl 2):2-12.

3. Neu H. The crisis in antibiotic resistance. Science. 1992;257:1064-1073.

4. Wildemauwe C, Godard C, Verschraegen G, Claeys G, Duyck MC, de Beenhouwer H, Vanhoof R. Ten years phage typing of Belgian clinical methicillin-resistant Staphylococcus aureus isolates (1992-2001). J Hosp Infect. 2004;56:1621.

5. Cordova SP, Heath $\mathrm{CH}$, McGechie DB, Keil AD, Beers MY, Riley TV. Methicillin-resistant Staphylococcus aureus bacteraemia in Western Australian teaching hospitals, 19971999: risk factors, outcomes and implications for management. J Hosp Infect. 2004;56:22-28.

6. Ayliffe G. The progressive intercontinental spread of methicillin-resistant Staphylococcus aureus. Clin Infect Dis. 1997;24(Suppl.1):S74-9.

7. Michel M, Gutmann L. Staphylococcus aureus resistente a la meticilina y enterococos resistentes a la vancomicina: realidades y posibilidades terapeuticas. The Lancet. 1997;31:312-317.

8. Franchi D, Climo MW, Wong AHM, Edmond MB, Wenzel RP. Seeking vancomycin resistant Staphylococcus aureus among patients with vancomycin - resistant Enterococci. Clin Infect Dis. 1999;29:1566-1568.

9. French G. Enterococci and vancomycin resistance. Clin Infect Dis. 1998;27 (Suppl 1):S75 -83.

10. Phillips I. Lessons from the past : a personal view. Clin Infect Dis. 1998;27 (suppl 1):S2-4.

11. Kernodle DS, Classen DC, Stratton ChW, Kaiser AB. Association of borderline oxacillin susceptible strains of Staphylococcus aureus with surgical wound infections. J Clin Microbiol. 1998;36:219-222.

12. Velazco E, Nieves B, Araque M, Calderas Z. Epidemiología de infecciones nosocomiales por Staphylococcus aureus en una unidad de alto riesgo neonatal. Enferm Infecc Microbiol Clin. 2002;20:321-325.

13. Rubio M, Romero J, Corral O, Roca V, Picazo J. Bacteriemia por Staphylococcus aureus: análisis de 311 episodios. Enferm Infecc Microbiol Clin. 1999; 17:56-64.

14. Jones M, Mayfield D, Thornsberry C, Karlowsky J, Sahm D, Peterson D. Prevalence of oxacillin resistance in Staphylococcus aureus among inpatients and outpatients in the United States during 2000. Antimicrob Agents Chemother. 2002;46:3104-3105.

15. Blanc D, Petignat C, Moreillon P, Entenza J, Eisenring M, Kleiber H, Wenger A, Troillet N, Blanc C, Francioli P. Unusual spread of a penicillin-susceptible methicillin-resistant Staphylococcus aureus clone in a geographic area of low incidence. Clin Infect Dis. 1999;29:1512-1518.

16. Sakoulas G, Gold H, Venkataraman L, Degirolami P, Eliopoulos G, Qian Q. Methicillin-resistant Staphylococcus aureus. Comparison of susceptibility testing methods and analysis of $m e c A$-positive susceptible strains. J Clin Microbiol. 2001;39:3946-3951. 
17. Fluit AC, Visser MR, Schmitz FJ. Molecular detection of antimicrobial resistance. Clin Microbiol Rev. 2001;14:836871.

18. Spratt BG. Resistance to antibiotics mediated by target alterations. Science. 1994;264:388-93.

19. Rivera JA. Antibiotic resistance, public health problem. An Med Asoc Med Hosp ABC. 2003;48:42-47.

20. Garza R, Vilchis G, Hernández L, Perea L. Estreptograminas: un modelo interesante para hacerle frente a la resistencia bacteriana. Lab-acta. 2004;16:59-65.

21. Martínez JL, Baquero F. Mutation frequencies and antibiotic resistance. Antimicrob Agents Chemother. 2000;44:17711777.

22. Guevara JM, Sánchez I, Mezarina E, Villagómez Z, Velarde N. Staphylococcus aureus resistente a la oxacilina en el Hospital Carrión. V Congreso Peruano de Enfermedades Infecciosas y Tropicales, 43, 1998.

23. Sánchez H, Carrillo L, Quispe M, Godoy A. Resistencia antibiótica de estafilococos en el Hospital Arzobispo Loayza de Lima, Perú. Boletín de la Sociedad Peruana de Enfermedades Infecciosas y Tropicales. 1998;7:9-10.

24. Siguas A, Salazar NA, Salazar A, Velásquez E, Villa Z, Siguas F. Susceptibilidad del Staphylococcus aureus en un servicio de medicina. V Congreso Peruano de Enfermedades Infecciosas y Tropicales, 1998: 27.

25. Vásquez H, Luccheti A. Sensibilidad del Staphylococcus aureus en cultivos de heridas, esputo y úlceras, HNERM, 1997. V Congreso Peruano de Enfermedades Infecciosas y Tropicales, 1998: 28.

26. Luján D. Evaluación de Staphylococcus aureus multirresistente en pacientes hospitalizados en el Instituto de Enfermedades Neoplásicas. Rev Per Enferm Infecc Trop. 2003;2:10-13.

27. Cornejo M, Azpilcueta A, Zea E, del Carpio J, Núñez D, Muñoz E. Staphylococcus aureus resistentes a oxacilina en el servicio de medicina del HNSA. V Congreso Peruano de Enfermedades Infecciosas y Tropicales, 1998: 22.
28. Mendoza C, Ballón J, de Los Ríos J, Velásquez R. Staphylococcus aureus meticilino resistente (MRSA) : colonización y susceptibilidad en pacientes y personal de salud de un hospital de referencia. Diagnóstico. 2001;40:149156.

29. Mendoza CA, Velásquez R, Mercado L, Ballón J, Maguiña C. Susceptibilidad antimicrobiana de Staphylococcus aureus sensible, con sensibilidad «borderline» y resistentes a la meticilina. Rev Med Hered. 2003;14:181-185.

30. Ubukata K, Yamashita N, Gotoh A, Cono M. Purification and characterization of aminoglycoside-modifying enzymes from Staphylococcus aureus and Staphylococcus epidermidis. Antimicrob Agents Chemother. 1984;25:754-759.

31. Hooper D. Fluoroquinolone resistance among gram-positive cocci. Lancet. 2002;2:530-538.

32. Sieradzki K, Roberts R, Haber S, Tomasz A. The development of vancomycin resistance in a patient with methicillin - resistant Staphylococcus aureus infection. N Eng J Med. 1999;340:517-523.

33. Smith T, Pearson M, Wilcox K, Cruz C, Lancaster M, Robinson-Dunn B, Tenover F, Zervos M, Band J, White E, Jarvis W. Emergence of vancomycin resistance in Staphylococcus aureus. N Eng J Med. 1999;340:493-501.

34. Centers for Disease Control. Staphylococcus aureus resistant to vancomycin-United States, 2002. MMWR 2002;51:565507.

Manuscrito recibido el 14 de marzo de 2006 y aceptado para publicación el 14 abril de 2006.

Correspondencia:

Blgo. Daniel Angel Luján Roca

Av. Garcilaso de la Vega 334 Dpto. 301

Urb. La Colonial. Callao, Perú

Correo-e:d_lujan@starmedia.com 